\title{
In Endothelial Cells, the Activation or Stimulation of Soluble Guanylyl Cyclase Induces the Nitric Oxide Production by a Mechanism Dependent of Nitric Oxide Synthase Activation
}

Ariane Migliato Martinelli, Carla Nascimento dos Santos Rodrigues, Thiago Francisco de Moraes, Gerson Jhonatan Rodrigues.

Departamento de Ciências Fisiológicas - Universidade Federal de São Carlos, São Carlos, SP, Brasil.

Received October 29, 2017; Revised, January 12, 2018; Accepted, January 22, 2018; Published, January 22, 2018.

\begin{abstract}
Purpose. In endothelial cells, investigate if the soluble guanylate cyclase (sGC) activation or stimulation is able to potentiate the relaxation in vessels. Methods. Aortic and coronary rings with and without endothelium were placed in a myograph and cumulative concentration-effect curves for DETA-NO or ataciguat were performed. Nitric oxide (NO) were measured by fluorescence or by selective electrode in human umbilical endothelial cells (HUVECs) in response to some treatments, including ataciguat, 8-BrcGMP and A23187. Results. The presence of the endothelium potentiated the relaxation induced by DETA$\mathrm{NO}$ in aortic and coronary rings. In addition, in aortic rings the endothelium potentiated the relaxation induced by ataciguat. In the presence of nitric oxide synthase (NOS) inhibitor, the endothelium effect was abolished to DETA-NO or ataciguat, in both vessels. Ataciguat, 8-Br-cGMP and A23187 were able to induce NO production in HUVECs cells. In the presence of NOS inhibitor, the NO production induced by ataciguat and 8-Br-cGMP was abolished. Conclusions. Our results suggest that in aortic and coronary rings the endothelium potentiates the relaxation induced by activation or stimulation of $\mathrm{sGC}$ through a mechanism dependent of NOS activation.
\end{abstract}

This article is open to POST-PUBLICATION REVIEW. Registered readers (see "For Readers") may comment by clicking on ABSTRACT on the issue's contents page.

\section{INTRODUCTION}

The vascular endothelium has an important protective role against cardiovascular disease, by release endothelium factor, which modulates vascular tonus, inflammation, and coagulation and the release of nitric oxide (NO) has a central role in this protection $[1,2]$. NO induces vasodilation by different mechanisms in order to decrease the cytoplasmic calcium concentration $\left[\mathrm{Ca}^{2+}\right]$ in vascular smooth muscle cells [3].

Within the muscle cell, NO interacts with iron from the heme group of the soluble guanylate cyclase enzyme (sGC), resulting in alteration of its conformation, making it active. The soluble guanylate cyclase enzyme catalyzes the release of two phosphate groups from the guanosine triphosphate (GTP) molecule, resulting in the production of cyclic guanosine monophosphate (cGMP) [4]. The cGMP activates cGMPdependent protein kinase (PKG), which phosphorylates a number of cellular proteins and may promote relaxation due to decreased $\mathrm{Ca}^{2+}$ influx, inhibition of release and / or increased $\mathrm{Ca}^{2+}$ storage in the sarcoendoplasmic reticulum
[5]. Therefore, cGMP plays a key role in the regulation of cardiovascular homeostasis, including smooth muscle relaxation, platelet inhibition, and vascular growth and differentiation [6].

Soluble guanylyl cyclase (sGC) is an important part of the NO/cGMP pathway, is expressed in the cytoplasm of almost mammalian cells, and mediates various functions, such as inhibition of aggregation platelet, smooth muscle relaxation, vasodilatation, signal transduction in neurons and immunomodulation [7]. The activation or stimulation of $\mathrm{sGC}$ results in increasing of cGMP levels in cells and finally causes relaxation in smooth muscle cells of the cardiovascular systems [8]. Soluble guanylyl cyclase activators are effective to induce relaxation in smooth muscle, therefore some of them have been used on clinical practice for pulmonary hypertension, congestive heart failure

Corresponding Author: Gerson Jhonatan Rodrigues, PhD, Departamento de Ciências Fisiológicas - Laboratório de Farmacologia, Rodovia Washington Luís, km 235 - SP-310, São Carlos - São Paulo - Brasil, CEP 13565-905; E-mail: gerson@ufscar.br 


\section{ABBREVIATIONS}

Acetylcholine (Ach) (PubChem CID:6060); Angiotensin II human (Ang II) (PubChem CID: 16211177); Ataciguat (PubChem CID: 213037); bromoguanosine 3',5'-cyclic monophosphate (8Br-cGMP) (PubChem CID: 104767); Calcimycin (A23187) (PubChem

CID:40486);

Diethylenetriamine/nitric oxide (DETA- NO) (PubChem CID 4518); L-Name (PubChem CID: 39836); Phenylephrine (Phe) (PubChem CID: 5284443); 4,5-diaminofluorescein diacetate (DAF-2DA); Verapamil (PubChem CID: 62969)

and erectile dysfunction [9]. The sGC activity is modulated by $\mathrm{sGC}$ stimulator or activator. Stimulation of sGC is dependent of its heme portion oxidative status, ferrous heme $\left(\mathrm{Fe}^{2+}\right)$ oxidative state is essential for it activation. Nitric oxide is a sGC stimulator and is a poor ligand of ferric heme $\left(\mathrm{Fe}^{3+}\right)$ [10, 11]. However, the activator like ataciguat (HMR 1766) bind to sGC when the iron is in the ferrous heme $\left(\mathrm{Fe}^{2+}\right)$, ferric heme $\left(\mathrm{Fe}^{3+}\right)$ state or even without this grouping $[12,13]$. Thus, the activator is able to activate the oxidized form of sGC [14-16].

The vasodilator effect of ataciguat has been determined previously [17]. In endothelial cells, the activation of sGC is able to induce the cGMP accumulation and induces the NO production, as demonstrated previously to activation induced by YC-1, which is a direct activator of sGC [18]. However, the endothelium modulatory effect on vascular relaxation induced by a newer sGC activator (ataciguat) and to an endogenous sGC stimulator (as the NO) have not been verified. Thus, the aim of this study was investigate if in endothelial cell the cGMP accumulation induced by a sGC stimulator or activator are able to modulate the vascular relaxation, as well investigate the mechanism of this effect.

\section{METHODS}

\section{Experimental animals}

Male Wistar rats were used weighing 180- $200 \mathrm{~g}$. Animals were maintained on a light-dark cycle with free access to both food (standard rat chow) and water.

\section{Vascular Reactivity Studies Aortic vascular reactivity}

Male Wistar rats were anesthetized with isoflurane and after euthanized by decapitation and the thoracic aortas were isolated. Aortic rings,
$3 \mathrm{~mm}$ in length, were placed in bath chambers $(5$ $\mathrm{mL}$ ) for isolated organs containing Krebs solution at $37^{\circ} \mathrm{C}$, continuously bubbled with $95 \% \mathrm{O}_{2}$ and $5 \% \mathrm{CO}_{2}, \mathrm{pH} 7.4$, in an isometric myograph (Mulvany-Halpern-model 610 DMT-USA, Marietta, GA) and recorded by a PowerLab8/SP data acquisition system (ADInstruments Pty Ltd., Colorado Springs, $\mathrm{CO}$ ).

The aortic rings were submitted to a tension of $1.5 \mathrm{~g}$, which was readjusted every $15 \mathrm{~min}$ throughout a 60 min equilibration period before the addition of the given drug. All the procedures were in accordance with the Animal Care and Use Committee of the Federal University of São Carlos (CEUA n ${ }^{\circ}$ 1626101216).

Endothelial integrity was assessed by the degree of relaxation induced by $1 \mu \mathrm{mol} / \mathrm{L}$ acetylcholine after contraction of the aortic ring by phenylephrine $(0.1 \mu \mathrm{mol} / \mathrm{L})$. The ring was discarded if relaxation with acetylcholine was lower than $80 \%$. After the endothelial integrity test, aortic rings were pre-contracted with phenylephrine $(0.1 \mu \mathrm{M})$ and then concentrationeffect curves to ataciguat or DETA-NO were done. Also to relaxation induced by ataciguat or DETA-NO, the endothelium was mechanically removed by gently rolling the lumen of the vessel on a thin wire. Endothelial integrity was assessed by relaxation induced by $1 \mu \mathrm{mol} / \mathrm{L}$ acetylcholine after contraction of the aortic ring by phenylephrine $(0.1 \mu \mathrm{mol} / \mathrm{L})$. The aortic rings were discarded if there was any sign of relaxation.

Aortic rings with endothelium were treated for 30 min with L-Name $100 \mu \mathrm{M}$, a non-specific NOS inhibitor. After incubation, aortic rings were pre-contracted with phenylephrine $(0.1 \mu \mathrm{M})$ and concentration-effect curves to ataciguat or DETANO were constructed. The maximum relaxant effect (ME) and the potency values $\left(\mathrm{pD}_{2}\right)$ were analyzed. Each experiment was performed on rings prepared from different rats.

\section{Coronary vascular reactivity}

Male Wistar rats were anesthetized with isoflurane and after euthanized by decapitation. Septal coronary arteries $(250-350 \mu \mathrm{m}$ in diameter) were carefully isolated and placed in freshly prepared ice-cold Krebs-Henseleit solution (KHS) that contained (mM): $\mathrm{NaCl} 118$; $\mathrm{KCl} 4.7 ; \quad \mathrm{NaHCO} 3 \quad 25 ; \quad \mathrm{CaCl} 2 \cdot 2 \mathrm{H} 2 \mathrm{O}$ 2.5; KH2PO4 1.2; $\mathrm{MgSO} 4 \cdot 7 \mathrm{H} 2 \mathrm{O}$ 1.2; EDTA 0.01; and glucose 11 . The coronary artery was cut into segments ( $2 \mathrm{~mm}$ in length) and were placed in bath chambers $(5 \mathrm{~mL})$ at $37^{\circ} \mathrm{C}$, continuously 
bubbled with $95 \% \mathrm{O}_{2}$ and $5 \% \mathrm{CO}_{2}, \mathrm{pH} 7.4$, in an isometric myograph (Mulvany-Halpern-model 610 DMT-USA, Marietta, GA) and recorded by a PowerLab8/SP data acquisition system (ADInstruments Pty Ltd., Colorado Springs, CO). The segments were washed with KHS and allowed to equilibrate for $30 \mathrm{~min}$. The vessel contractility was then tested by exposure to a high- $\mathrm{K}^{+}$(KCL: $\left.120 \mathrm{mM}\right)$ solution. Integral vessels were considered those that presented relaxation for acetylcholine higher than $80 \%$. The vessels that were inferior to those reported were discarded. The endothelium was mechanically removed by gently rolling the lumen of the vessel on a thin wire.

Coronary rings with endothelium were treated for 30 min with L-Name $100 \mu \mathrm{M}$, a non-specific NOS inhibitor. In all experiments, the coronary rings were pre-contracted with serotonin $(10 \mu \mathrm{M})$ and concentration-effect curves to DETA-NO were performed. The maximum relaxant effect (ME) and the potency values (pD2) were analyzed. Each experiment was performed on rings prepared from different rats.

\section{Cell Culture}

Immortalized human umbilical endothelial cells (HUVEC) were grown in DMEM (Inlab) supplemented with $10 \%$ of fetal calf serum, antibiotics and antimycotics. Cultures were maintained at $37 \pm 2{ }^{\circ} \mathrm{C}$ in $5 \% \mathrm{CO}_{2}$ atmosphere. The cells in confluence of 80 to $90 \%$ were trypsinised, centrifuged at $1200 \mathrm{rpm}$ during 5 minutes, and then plated in 96-well plates (TPP).

\section{Nitric Oxide (NO) Measurements By fluorescence}

HUVEC were placed in 96 well plates at the concentration of $5 \times 10^{4}$ cells per well. The plates were incubated for 24 hours in humidified incubator containing $5 \% \mathrm{CO} 2,37^{\circ} \mathrm{C}$. After 24 hours, the treatment was removed and the plates were gently washed with Phosphate Buffer Saline (PBS). The detection of intracellular NO was performed by incubation with selective fluorescent probe 4,5-diaminofluorescein (DAF$2 \mathrm{~T}-10 \mu \mathrm{M}$ ) during 30 minutes, to react with dinitrogen trioxide (N2O3) (oxidation product of NO) and produces the fluorescent compound DAF-2T [19]. The reading was held in SpectraMax GeminiXS fluorometer (Molecular Devices) at $435 \mathrm{~nm}$ excitation and $538 \mathrm{~nm}$ emission wavelength pair, respectively.

\section{By electrode}

The NO quantification was performed by a selective electrode for NO (InNO-T-II, Inovative Instruments, Inc.) indirectly by the quantification of nitrite $\left(\mathrm{NO}_{2}{ }^{-}\right)$which is a NO stable metabolite. According to the manufacturer, the InNO-T-II electrode has a minimum detection limit of $0.01 \mathrm{nM}$. In a constant shaking vessel was added 1 $\mathrm{mL}$ of working solution which was composed of purified deionized water in a Milli- ${ }^{\circledR}$ system, $1 \mathrm{M}$ sulfuric acid and $90 \mathrm{mM}$ potassium iodide (working solution). In this solution the nitrite reduction in NO (equation 1) occurs, which can be detected by the electrode.

$$
2 \mathrm{NO}_{2}{ }^{-}+2 \mathrm{I}^{-}+4 \mathrm{H}^{+} \rightarrow 2 \mathrm{NO}+\mathrm{I}_{2}+2 \mathrm{H}_{2} \mathrm{O}(1)
$$

Endothelial cells were plated in 6-well plates at a concentration of $4 \times 10^{5}$ cells per well. After 24 hours of plating, cells were treated with PBS, 8Br-GMPc at concentration: 1,10 and $100 \mu \mathrm{M}$; A23187 $10 \mu \mathrm{M}$ and L-Name $1 \mu \mathrm{M}$. For reading, the supernatant was removed from each well and placed in the shaker vessel together with the working solution where the electrode was immersed. The readings were performed at the time 180 minutes. A calibration curve to nitrite was performed before each experiment.

\section{STATISTICAL ANALYSIS}

Statistical analysis of the results was performed using GraphPad Prism version 3.0. Statistical significance was tested by ANOVA one way (post hoc test: Newman-Keuls). Data are expressed as mean \pm S.E.M. In each set of experiments, $n$ indicates the number of rats studied. Values of $p<$ 0.05 were considered significant.

\section{RESULTS}

In aortic rings, the relaxation induced by ataciguat (Figure 1A) and DETA-NO (Figure 1B) was potentiated in the presence of endothelial cells. In aortic rings without endothelium or with endothelium but in the presence of non-specific NOS inhibitor (L-Name), the relaxation to ataciguat (Figure 1A) and DETA-NO (Figure 1B) was impaired. In coronary rings, the presence of endothelial cells also potentiated the relaxation to DETA-NO and the non-specific NOS inhibitor (L-Name) excluded the endothelium effect (Figure 1C). 


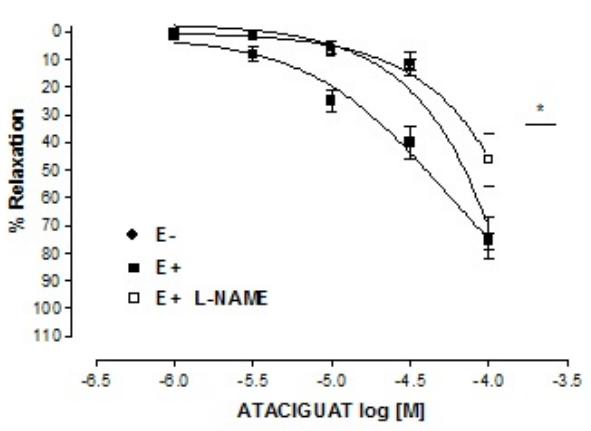

A

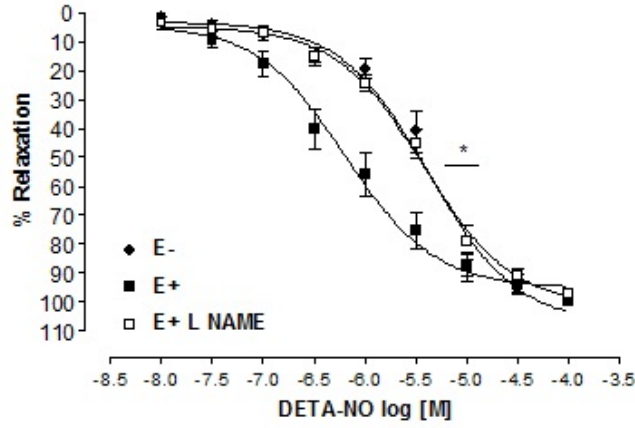

B

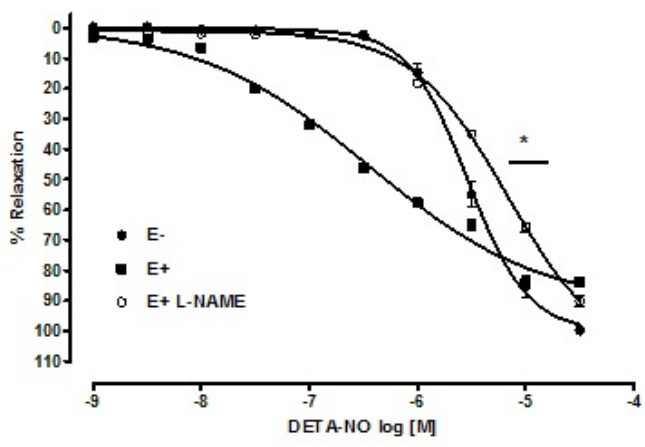

C

Figure 1. Concentration-effect curve to Ataciguat or DETA-NO in aortic rings or coronary rings, with (E+) and without (E-) endothelium and in the presence of L-NAME. A. Concentration-effect curve to Ataciguat in aortic rings with (E+) and without (E-) endothelium and in the presence of non-specific NOS inhibitor (L-NAME) B. Concentration-effect curve to DETA-NO in aortic rings with $(\mathrm{E}+)$ and without $(\mathrm{E}-)$ endothelium and in the presence of non-specific NOS inhibitor (L-NAME). C. Concentration-effect curve to DETA-NO in coronary rings with (E+) and without (E-) endothelium and in the presence non-specific NOS inhibitor (L-NAME). Values are the means \pm S.E.M. of the number of experiments on preparations obtained from different animals. * Indicates difference between pD2 E+ vs. pD2 E-, and pD2 E+ vs. pD2 E+ $(+)$ L-NAME $(\mathrm{p}<0.05)$.

To verify whether endothelium potentiated the relaxation to ataciguat by a mechanism dependent of NO production, we performed experiments in isolated HUVEC cells. In Figure 2, the treatment of HUVECs by ataciguat for 30 minutes was able to increase NO production in endothelial cells as compared to the untreated control (Control: $5.36 \pm$ $0.33 \mathrm{FI}, \mathrm{n}=5$; Ataciguat $0.1 \mu \mathrm{M}: 10.56 \pm .0 .50 \mathrm{FI}$, $\mathrm{n}=5$; Ataciguat $1 \mu \mathrm{M}$ : $9.14 \pm 0.19$ FI, $\mathrm{n}=5$; Ataciguat $10 \mu \mathrm{M}: 8.52 \pm 0.21 \mathrm{FI}, \mathrm{n}=5, \mathrm{p}<0.001$ ). In addition, the treatment with the lowest concentration of ataciguat $(0.1 \mu \mathrm{M})$ was able to promote a two-fold increase over PBS, indicating that the activation of sGC in the endothelial cells by ataciguat is able to induces NO production.

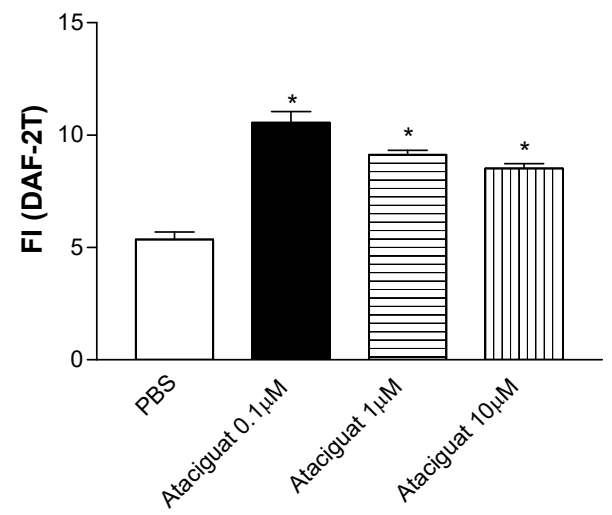

Figure 2. Intracellular NO quantification in HUVEC 


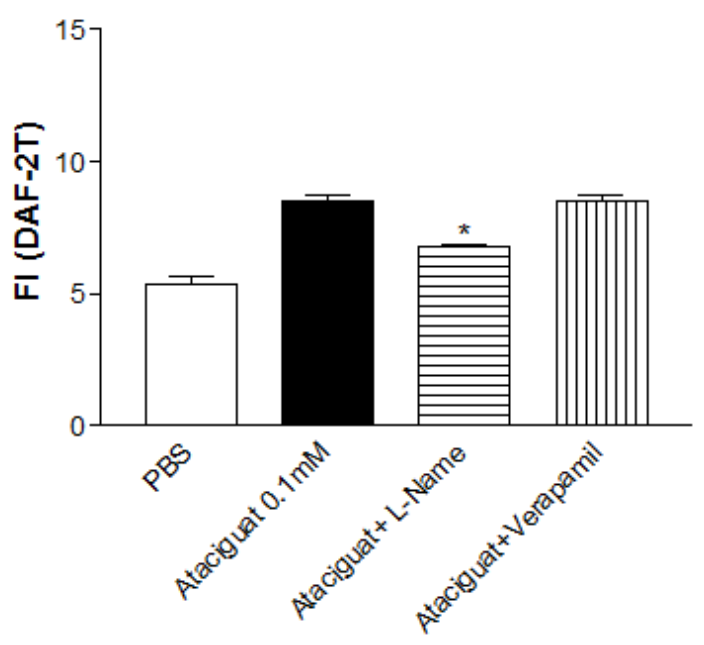

Figure 3. Intracellular NO quantification in HUVEC

Values are means \pm S.E.M of fluorescence intensity obtained on HUVEC cells after 30 minutes of treatment with PBS and Ataciguat. * Indicates difference between Ataciguat $0.1 \mu \mathrm{M}$ vs PBS; Ataciguat $1 \mu \mathrm{M}$ vs PBS and Ataciguat $10 \mu \mathrm{M}$ vs PBS ( $<<0.001)$.

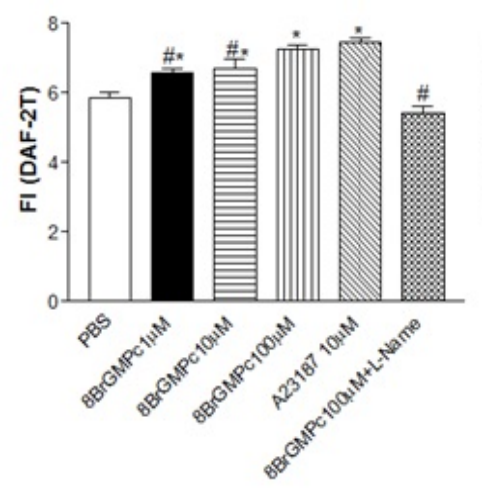

A

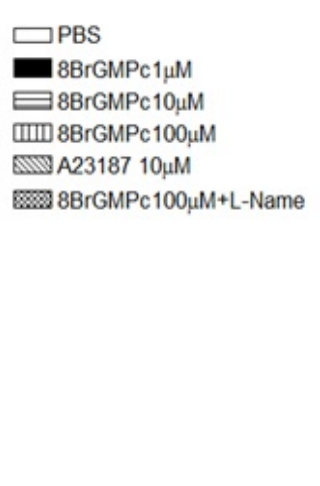

Values are means \pm S.E.M of the fluorescence intensity obtained on HUVEC cells after 30 minutes of treatment with PBS, Ataciguat, L-Name and Verapamil.* Indicates difference between Ataciguat vs Ataciguat + LName $(\mathrm{p}<0.001)$.

Figure 4. NO quantification in HUVEC - A. Quantification of NO by fluorescent intensity of DAF, before and after 30 min treatment with PBS, 8-Br-GMPc, A23187 and L-Name. Values are means \pm S.E.M. fluorescence intensity obtained on HUVEC cells after 30 minutes of treatment with PBS, 8-Br-GMPc and A23187. * Indicates difference between PBS vs 8-Br-GMPc $1 \mu \mathrm{M}, 10 \mu \mathrm{M}$ and $100 \mu \mathrm{M}(\mathrm{p}<0.05)$. \# indicates difference between $\mathrm{A} 23187 v s 8-\mathrm{Br}-$ GMPc $1 \mu \mathrm{M}$ and $10 \mu \mathrm{M}$ and 8-Br-GMPc $100 \mu \mathrm{M} v s$-Br-GMPc $100 \mu \mathrm{M}+\mathrm{L}-\mathrm{Name}(\mathrm{p}<0.05)$. B. Quantification of NO by using electrode after 30 minutes treatment with PBS, 8-Br-GMPc, A23187 and L-Name. * Indicates statistical difference between PBS $v s$ 8-Br-GMPc $1 \mu \mathrm{M}, 10 \mu \mathrm{M}$ and $100 \mu \mathrm{M}(\mathrm{p}<0.05)$. \# indicates difference between A23187 $v s$

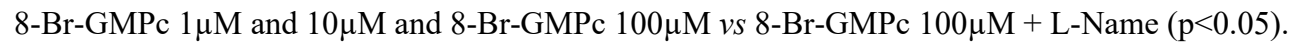


To investigate the mechanism by which NO production is increased, the treatment was performed with ataciguat in the presence of nonspecific NOS inhibitor (L-Name) or verapamil, a $\mathrm{Ca}^{2+}$ channel blocker. How can be verified at Figure 3, NOS inhibition decreased the ataciguat effect (Control: $5.36 \pm 033, \mathrm{n}=5$; Ataciguat $0.1 \mu \mathrm{M}: 8.52 \pm 0.20 \mathrm{FI}, \mathrm{n}=5$; Ataciguat $+\mathrm{L}-\mathrm{Name}$ : $6.75 \pm 0.14 \mathrm{FI}, \mathrm{n}=5$ ). However, in the presence of verapamil the ataciguat effect was not modified (Ataciguat + Verapamil: $8.54 \pm 0.21 \mathrm{FI}, \mathrm{n}=5$ ). It suggest that the mechanism of NO production induced by ataciguat is not dependent of $\mathrm{Ca}^{2+}$ influx.

To investigate if the accumulation of cGMP is able to induce NO production in endothelial cells, we have used the 8-Br-GMP, which is a cGMP analogue. As can be verified at Figure 4 the $8-\mathrm{Br}$ cGMP induced NO production in HUVEC cells. To confirm this effect we have used two methods of NO quantification, by fluorescence or by selective NO electrode. In all concentration used the 8-Br-GMP induced elevation in NO concentration, compared to control in the quantification made by fluorescence (PBS: 5.85 $\pm 0.17 \mathrm{FI} ; \mathrm{n}=5 ; 8$-Br-GMPc $1 \mu \mathrm{M}: 6.57 \pm 0.11$ FI, $\mathrm{n}=5$; 8-Br-GMPc $10 \mu \mathrm{M}$ : $6.71 \pm 0.25 \mathrm{FI}, \mathrm{n}=5$; $8=$ Br-GMPc $100 \mu \mathrm{M}: 7.26 \pm 0.10 \mathrm{FI}, \mathrm{n}=5, \mathrm{p}<0.05$ ) and by electrode (PBS: $20.40 \pm 0.56 \mathrm{nM}, \mathrm{n}=5 ; 8$ Br-GMPc $1 \mu \mathrm{M}: 29.60 \pm 0.74 \mathrm{nM}, \mathrm{n}=5 ; 8$-BrGMPc $10 \mu \mathrm{M}: 30.90 \pm 0,82 \mathrm{nM}, \mathrm{n}=5 ; 8=\mathrm{Br}-$ GMPc $100 \mu \mathrm{M}$ : $36.80 \pm 0.60 \mathrm{nM}, \mathrm{n}=5, \mathrm{p}<0.05$ ). The 8-Br-cGMP at $100 \mu \mathrm{M}$ promoted a production of NO similar to A23187 (Fluorescence: 8-BrGMPc $100 \mu \mathrm{M}: 7.26 \pm 0.10 \mathrm{FI}, \mathrm{n}=5$; A23187: 7.46 $\pm 0.10 \mathrm{FI}, \mathrm{n}=5$ ) (Eletrode: 8-Br-GMPc $100 \mu \mathrm{M}$ : $36.80 \pm 0.60 \mathrm{nM}, \mathrm{n}=5 ;$ A23: $37.50 \pm 0.87 \mathrm{nM}$, $\mathrm{n}=5$ ) with no statistical difference. When treated with L-Name, the NO production was decreased (DAF-2T: 8-Br-GMPc $100 \mu \mathrm{M}: 7.26 \pm 0.10 \mathrm{FI}$, $\mathrm{n}=5$; 8 -Br-GMPc $100 \mu \mathrm{M}+$ L-Name: $5.43 \pm 0.17$ FI, $\mathrm{n}=5, \mathrm{p}<0.05$ ) (Eletrode: 8-Br-GMPc $100 \mu \mathrm{M}$ : $36.80 \pm 0.60 \mathrm{nM}, \mathrm{n}=5 ; 8-\mathrm{Br}-\mathrm{GMPc} 100 \mu \mathrm{M}+\mathrm{L}-$ Name: $21.10 \pm 0.68, \mathrm{n}=5, \mathrm{p}<0.05$ ).

\section{DISCUSSION}

The main finding of this study was that cGMP accumulation in endothelial cells induced by sGC activator or stimulator is able to induce NO production with consequent potentiation of vascular relaxation.

Our results showed that the presence of endothelium potentiated the relaxation induced by
sGC activator (ataciguat) and by stimulator (DETA-NO) and are in accordance to previous work, which showed that the presence of endothelial cells potentiated the vascular relaxation induced by $\mathrm{YC}-1$ that is an activation of sGC [18]. To investigate if this potentiation on relaxation is due to $\mathrm{NO}$ production, we have performed experiments in aortic and coronary rings with without endothelium and with endothelium in the presence of NOS inhibitor. In both vessels, the endothelium effect was abolished in the presence of NOS inhibitor, indicating that it effect is due to NO production.

Ataciguat is an improved sGC activator capable of activating the ferric heme-iron redox form of $\mathrm{sGC}$ that stimulate the production of cyclic GMP (cGMP), which induces a reduction in the concentration of cytosolic $\mathrm{Ca}^{2+}$ in smooth muscle cells with consequent vascular relaxation $[20,13]$. Its effect in vascular smooth muscle cells have been well described, however, in endothelial cells have not been described extensively, as well the cGMP effect.

In isolated endothelial cells, we have verified that the activation of sGC and the 8-Br-cGMP are able to induce NO production. These results was confirmed by two different methods of $\mathrm{NO}$ quantification, including fluorescence (by using DAF-2DA) measuring the intracellular NO and by electrode measuring through conversion of nitrite (stable metabolite) to NO. Ataciguat is able to induce cGMP accumulation [21] which can activates NOS in endothelial cells, considering that the endothelium treatment with cGMP analogue also stimulated the NO production by NOS activation. Our results suggested that NO production is by a mechanism dependent of NOS activation, and independent of $\mathrm{Ca}^{2+}$ influx. In isolated aortic rings the endothelium potentiates the relaxation induced by sodium nitroprusside (NO donor), by a mechanism dependent of endothelium $\mathrm{Ca}^{2+}$ influx with consequent NOS activation, as verified previously [22]. Under reduction, the SNP release NO [22] and cyanide [23] and the compound DETA-NO release just $\mathrm{NO}$ and spontaneously [24]. Thus, DETA-NO is a better pharmacological tool to investigate the effect of NO in endothelial cells. In addition, our results indicated ataciguat reached the maximum NO production effect in HUVEC cells at $0.1 \mu \mathrm{M}$, considering that higher concentration (1.0 and $10 \mu \mathrm{M})$ does not increase the effect. These results suggest that low ataciguat concentration is able to induce NO production in endothelial cells. 
Our results suggest that NO itself may induce stimulation of its own synthesis in endothelial cells. Thus, these results contribute to one more effect of $\mathrm{NO}$ on modulation of endothelial function.

\section{CONCLUSION}

Our results suggest that the activation or stimulation of sGC in endothelial cells induces the NO production by a mechanism dependent of NOS activation, with consequent potentiation on vascular relaxation induced by activator or stimulator of sGC.

\section{ACKNOWLEDGMENTS}

This work was supported by grants from São Paulo Research Foundation (FAPESP grants: 2012/24477-8, 2014/01341-9) and National Counsel of Technological and Scientific Development (CNPq grant 478849/2013-3).

\section{REFERENCES}

1. Davel AP, Wenceslau CF, Akamine EH, Xavier FE, Couto GK, Oliveira HT, Rossoni LV. Endothelial dysfunction in cardiovascular and endocrine-metabolic diseases: an update. Braz J Med Biol Res. 2011; 44: 920-32.

2. Vanhoutte PM. Regeneration of the Endothelium in Vascular Injury. Cardiovasc Drugs Ther. 2010; 24: 299-03.

3. Ignarro LJ. Biological actions and properties of endothelium- derived nitric oxide formed and released from artery and vein. Circulation Research. 1989; 65: 1-21.

4. Rapoport RM, Murad F. Agonist-induced endothelium dependent relaxation in rat thoracic aorta may be mediated through cGMP. Circ. Res. 1983; 52: 352-57.

5. Gewaltig J, Mangasser-Stephan K, Gartung C, Biesterfeld S, Gressner AM. Association of polymorphisms of the transforming growth factorb1 gene with the rate of progression of HCVinduced liver fibrosis. Clin Chem Acta. 2002; 316: 83-94.

6. Schmidt HH, Schmidt PM, Stasch JP. NO- and haem-independent soluble guanylate cyclase activators. Handb Exp Pharmacol. 2009; 191: 30939 .

7. Collier J, Vallance P. Second messenger role for NO widens to nervous and immune systems. Trends Pharmacol Sci. 1989; 10: 427-31.

8. Potter LR. Guanylyl cyclase structure, function and regulation. Cell Signal. 2011; 23: 1921-26.
9. Buechler WA, Nakane M, Murad F. Expression of soluble guanylate cyclase activity requires both enzyme subunits. Biochem Biophys Res Commun. 1991; 174: 351-7.

10. Stone JR, Marletta MA. Soluble Guanylate Cyclase from Bovine Lung: Activation with Nitric Oxide and Carbon Monoxide and Spectral Characterization of the Ferrous and Ferric States. Biochemistry. 1994; 33: 5636 - 40.

11. Stone JR, Sands RH, Dunham WR, Marletta MA. Spectral and ligand-binding properties of an unusual hemoprotein, the ferric form of soluble guanylate cyclase. Biochemistry. 1996; 35: 3258 62.

12. Stasch JP, Pacher P, Evgenov OV. Soluble guanylate cyclase as an emerging therapeutic target in cardiopulmonary disease. Circulation. 2011; 123: 2263-73.

13. Surmeli NB, Marletta MA. Insight into the rescue of oxidized soluble guanylate cyclase by the activator cinaciguat. Chembiochem, 2012; 7: 977-981.

14. Schindler U, Strobel H, Schönafinger K, Linz W, Löhn M, Martorana PA, Rütten H, Schindler PW, Busch AE, Sohn M, Töpfer A, Pistorius A, Jannek C, Mülsch A. Biochemistry and pharmacology of novel anthranilic acid derivatives activating hemeoxidized soluble guanylyl cyclase. Molecular Pharmacology 2006; 69: 1260-68.

15. Zhou Z, Pyriochou A, Kotanidou A., et al. Soluble guanylyl cyclase activation by HMR-1766 (ataciguat) in cells exposed to oxidative stress. American Journal of Physiology.Heart and Circulatory Physiology. 2008; 295: 1763-71.

16. Stasch JP, Schmidt P, Alonso-Alija C, et al. NOand haemindependent activation of soluble guanylyl cyclase: molecular basis and cardiovascular implications of a new pharmacological principle. Br J Pharmacol. 2002; 136: 773-83.

17. Schäfer A, Fraccarollo D, Werner L, Bauersachs J. Guanylyl cyclase activator ataciguat improves vascular function and reduces platelet activation in heart failure.Pharmacological Research. 2010; 62: 432-38.

18. Wohlfart P, Malinski T, Ruetten H., Schindler U, Linz W. Schoenafinger, K., Strobel,H. Wiemer, G. Release of nitric oxide from endothelial cells stimulated by YC-1, an activator of soluble guanylyl cyclaseBr J Pharmacol. 1999; 128: 131622.

19. Nakatsubo N, Kojima H, Kikuchi K, Nagoshi H, Hirata Y, Maeda D, Imai Y, Irimura T, Nagano T. Direct evidence of nitric oxide production from bovine aortic endothelial cells using new fluorescence indicators: diaminofuoresceins. FEBS Letters. 1998; 42:263-66.

20. Hoffmann LS, Schmidt PM, Keim Y, Hoffmann C, Schmidt HH, Stasch JP. Fluorescence 
dequenching makes haem-free soluble guanylate cyclase detectable in living cells. PLoS One. 2011; 6:235-96.

21. Nossaman B, Pankey E, Kadowitz P. Stimulators and activators of soluble guanylate cyclase: review and potential therapeutic indications. Crit Care Res Pract. 2012: 290805-17.

22. Bonaventura D, Lunardi CN., Rodrigues G.J, Neto MA, Bendhack, LM. A novel mechanism of vascular relaxation induced by sodium nitroprusside in the isolated rat aorta. Nitric Oxide. 2008; 18:287-95.

23. Arnold WP, Longnecker, DE, Epstein RM. Photodegradation of sodium nitroprusside: biologic and cyanide release. Anesthesiology. 1984; 61:254-60.

24. Schyvens CG, Cowden WB, Zhang Y, McKenzie KU, Whitworth JA. Hemodynamic effects of the nitric oxide donor DETA/NO in mice. Clin Exp Hypertens. 2004; 26:525-35. 